\title{
MOMENT PROBLEM FOR A BOUNDED REGION ${ }^{1}$
}

\section{B. HEDGE}

1. Introduction. In this paper a solution of the moment problem given by Hausdorff ${ }^{2}$ for a bounded interval is extended to any bounded region in euclidean $n$-space, under certain conditions on polynomial expansions over the region. The resulting solution is valid for the $n$-dimensional sphere, and includes the Hausdorff case as well as the known conditions on the "class" of Fourier and FourierStieltjes series. ${ }^{3}$

2. Definitions and notation. Let $n$ be a positive integer, fixed but arbitrary. $R^{n}$ will denote the euclidean $n$-space, $(x)$ and $(y)$ will stand for $\left(x_{1}, x_{2}, \cdots, x_{n}\right)$ and $\left(y_{1}, y_{2}, \cdots, y_{n}\right)$, points of $R^{n}$, and $E$ a bounded, closed subset of $R^{n} . \nu, \tau, i, j, k$, and $s$, will be used for nonnegative integers, and $(k),(s)$, and so on, will denote ordered $n$-tuples of non-negative integers $\left(k_{1}, k_{2}, \cdots, k_{n}\right),\left(s_{1}, s_{2}, \cdots, s_{n}\right)$, and so on, $(k)=(s)$ will mean $k_{i}=s_{i}, i=1,2, \cdots, n$. (0) will mean $(0,0, \cdots, 0)$, $\left\{\mu_{(m)}\right\}$ will be a sequence of real numbers, and $\left\{U_{(k)}(x)\right\}$ and $\left\{V_{(k)}(x)\right\}$ will be two sequences of polynomials such that

$$
\begin{gathered}
U_{(0)}(x)=V_{(0)}(x)=\text { const. }, \\
\int_{E} U_{(k)}(x) V_{(s)}(x) d x=\left\{\begin{array}{l}
0,(k) \neq(s), \\
1,(k)=(s),
\end{array}\right.
\end{gathered}
$$

and by $\int_{E(y)} f(x, y) d \Phi(E)$ will be meant the Lebesgue-Stieltjes integral over $E$ of $f$ considered as a function of a point $(y) . B$ will be used for any Borel set with $B \subseteq E$.

If $f$ is integrable over $E$ we define

$$
\begin{gathered}
\Im(f, x) \simeq \sum_{(k)} A_{(k)} V_{(k)}(x), \quad A_{(k)}=\int_{E} f(x) U_{(k)}(x) d x, \\
S(x, y) \simeq \sum_{(k)} U_{(k)}(x) V_{(k)}(y) .
\end{gathered}
$$

Let $L_{\nu}$ for every $\nu$ be a partition of $R^{n}$ into two subsets, one closed and bounded. We write $(k) \in L_{\nu}$ to indicate that $(k)$ belongs to the

\footnotetext{
1 Presented to the Society, June 20, 1940.

${ }^{2} \mathrm{~F}$. Hausdorff, Momentprobleme für ein endliches Intervall, Mathematische Zeitschrift, vol. 16 (1923), pp. 220-248.

${ }^{3}$ See, for example, A. Zygmund, Trigonometrical Series, Monografje Matematyczne, vol. 5, Warsaw, 1935, pp. 79-86.
} 
bounded subset defined by $L_{\nu}$, and require that for every $(k)$ there exist a $\nu$ such that $(k) \in L_{\nu}$, and that $(k) \in L_{\nu}$ shall imply $(k) \in L_{\nu^{\prime}}$ for all $\nu^{\prime} \geqq \nu$. Now let

$$
\begin{aligned}
& S_{\nu}(x, y)=\sum_{(k) \in L_{\nu}} U_{(k)}(x) V_{(k)}(y), \\
& \Im_{\nu}(f, x)=\sum_{(k) \in L_{\nu}} A_{(k)} V_{(k)}(x)=\int_{E} S_{\nu}(x, y) f(y) d y .
\end{aligned}
$$

If $T:\left\|a_{i j}\right\|$ is any regular Toeplitz transformation, ${ }^{4}$ we write

$$
T \Im_{\nu}(f, x)=\int_{E} T S_{\nu}(x, y) f(y) d y=\int_{E} K_{\nu}(x, y) f(y) d y .
$$

If $P$ is a polynomial in $(x)$ we denote by $\mu_{(x)}(P)$ the expression resulting from the substitution of $\mu_{m_{1}, m_{2}}, \cdots, m_{n}$ for $x_{1}^{m_{1}} x_{2}^{m_{2}} \cdots x_{n}^{m_{n}}$ in $P$.

3. Moment problem. A solution of the moment problem for the set $E$ is given in the following theorem:

Theorem. Given $\left\{U_{(k)}(x)\right\},\left\{V_{(k)}(x)\right\},\left\{L_{\nu}\right\}$, and $T$ satisfying the conditions above, and such that $T S_{\nu}(x, y)=K_{\nu}(x, y) \geqq 0$ for all $(x),(y) \in E$, and all $\nu$, and such that for any $f$ integrable over $E$ $T \Im_{\nu}(f, x) \rightarrow f(x)$ for every $(x) \in E$ for which $f$ is continuous, and uniformly on $E$ if $f$ is continuous on $E$, then in order that a sequence $\left\{\mu_{(m)}\right\}$ be expressible in the form

$$
\mu_{m_{1}, m_{2}, \cdots, m_{n}}=\int_{E} x_{1}^{m_{1}} x_{2}^{m_{2}} \cdots x_{n}^{m_{n}} d \Phi(E),
$$

where $\Phi$ is completely additive, defined over at least all Borel sets of $R^{n}$, and with

(1) $\int_{E}|d \Phi(E)| \leqq M$,

(2) $\Phi(B) \geqq 0$,

(3) $\Phi(B)=\int_{B} \phi(x) d x$ and with

(3a) $\phi \in L_{E}^{p}, p>1$,

(3b) $\phi \in L_{E}$,

(3c) $|\phi| \leqq M$,

(3d) $\phi \in C_{E}$,

it is necessary and sufficient that

(1) $\int_{E}\left|\mu_{(y)}\left\{K_{\nu}(x, y)\right\}\right| d x \leqq M$ for all $\nu$,

(2) $\mu_{(y)}\left\{K_{\nu}(x, y)\right\} \geqq 0$ for all $(x) \in E$ and all $\nu$,

(3a) $\int_{E}\left|\mu_{(y)}\left\{K_{\nu}(x, y)\right\}\right|{ }^{p} d x \leqq M$ for all $\nu$,

${ }^{4}$ Zygmund, loc. cit., pp. 40-43. 
(3b) $\lim _{\nu, \tau \rightarrow \infty} \int_{E}\left|\mu_{(y)}\left\{K_{\nu}(x, y)\right\}-\mu_{(y)}\left\{K_{\tau}(x, y)\right\}\right| d x=0$,

(3c) $\left|\mu_{(y)}\left\{K_{\nu}(x, y)\right\}\right| \leqq M$ for all $(x) \in E$ and all $\nu$,

(3d) $\lim _{\nu, \tau \rightarrow \infty}\left|\mu_{(y)}\left\{K_{\nu}(x, y)\right\}-\mu_{(y)}\left\{K_{\tau}(x, y)\right\}\right|=0$ uniformly in $(x) \in E$.

The proof in each of the six cases closely parallels that of Hausdorff. ${ }^{2}$ The proof is given for case (1) to indicate the modifications:

Necessity. We have

$$
\begin{aligned}
\left|\mu_{(y)}\left\{K_{\nu}(x, y)\right\}\right| & =\left|\int_{E(y)} K_{\nu}(x, y) d \Phi(E)\right| \\
& \leqq \int_{E(y)} K_{\nu}(x, y)|d \Phi(E)| \\
\int_{E}\left|\mu_{(y)}\left\{K_{\nu}(x, y)\right\}\right| d x & \leqq \int_{E}\left\{\int_{E} K_{\nu}(x, y) d x\right\}|d \Phi(E)| \\
& \leqq C \int_{E}|d \Phi(E)| \leqq M
\end{aligned}
$$

for

$$
\begin{aligned}
K_{\nu}(x, y) & =\sum_{j=0}^{\infty} a_{\nu j} \sum_{(k) \in L_{j}} U_{(k)}(x) V_{(k)}(y) \\
\int_{E} K_{\nu}(x, y) d x & =\sum_{j=0}^{\infty} a_{\nu j} \sum_{(k) \in L_{j}} V_{(k)}(y) \int_{E} U_{(k)}(x) d x \\
& =\sum_{j=0}^{\infty} a_{\nu j} \leqq \sum_{j=0}^{\infty}\left|a_{\nu j}\right| \leqq C .
\end{aligned}
$$

Sufficiency. Let

$$
\begin{gathered}
\Phi_{\nu}(B)=\int_{B} \mu_{(y)}\left\{K_{\nu}(x, y)\right\} d x \\
\int_{E}\left|d \Phi_{\nu}(E)\right|=\int_{E}\left|\mu_{(y)}\left\{K_{\nu}(x, y)\right\}\right| d x \leqq M
\end{gathered}
$$

and, by a well known theorem of Helly, there is a subsequence $\left\{\Phi_{\nu^{\prime}}\right\}$ and a function $\Phi$ such that $\int_{E}|d \Phi(E)| \leqq M$ and $\Phi_{\nu^{\prime}}(B) \rightarrow \Phi(B)$, and also $\int_{E} V_{(k)}(y) d \Phi_{\nu^{\prime}}(E) \rightarrow \int_{E} V_{(k)}(y) d \Phi(E)$ whence $\mu_{(y)}\left\{V_{(k)}(y)\right\}$ $=\int_{E} V_{(k)}(y) d \Phi(E)$, and $\Phi$ is a solution.

4. Examples and conclusion. If $E$ is the unit sphere in $R^{n},\left\{U_{(k)}(x)\right\}$ and $\left\{V_{(k)}(x)\right\}$ may be taken as the normalized polynomials of Appell- 
$\operatorname{Didon}^{5}(k) \in L_{\nu}$ to mean $\sum_{i=1}^{n} k_{i} \leqq \nu$, and $T$ any $(C, r)$ with $r \geqq n+1 .^{6}$ In particular, for $n=1$ this reduces to the Hausdorff solution for the unit interval. If $E$ is the circumference of the unit circle we may set $U_{0}(x)=V_{0}(x)=(2 \pi)^{-1 / 2}$, and, for $k>0$,

$U_{2 k}(x)=V_{2 k}(x)=(\pi)^{-1 / 2} \cos k \theta, \quad U_{2 k-1}(x)=V_{2 k-1}(x)=(\pi)^{-1 / 2} \sin k \theta$

with $(s) \in L_{\nu}$ meaning $s \leqq 2 \nu, T$ any $(C, r)$ with $r \geqq 1 .^{7}$ Sequences $\left\{U_{(k)}(x)\right\}$ and $\left\{V_{(k)}(x)\right\}$ can be constructed by the Schmidt process for any bounded region in $R^{n}$. It would be interesting to know whether regular Toeplitz transformations of the type required for the present theorem exist in general.

\section{BROWN UNIVERSITY}

${ }^{5} \mathrm{P}$. Appell and J. Kampé de Fériet, Fonctions Hypergéométriques et Hypersphériques; Polynomes d'Hermite, Paris, 1926.

${ }^{6} \mathrm{~L}$. Koschmieder, Über die C-Summierbarkeit gewisser Reihen von Didon und Appell, Mathematische Annalen, vol. 104 (1931), pp. 387-402.

${ }^{7}$ L. Fejer's theorem. See, for instance, Zygmund, loc. cit., p. 45. 